\section{Kolonkarzinomvorsorge: Reicht es, nur ins Sigmoid zu schauen?}

Die vergleichsweise schnell und einfach durchzuführende Darmkrebsvorsorge mit dem flexiblen Sigmoidoskop halbiert die Mortalität distaler Karzinome, hat aber keinen Einfluss auf die Sterblichkeit proximaler Darmtumoren.

- Der Goldstandard der Prävention beim kolorektalen Karzinom ist die totale Koloskopie. Mit ihrer Hilfe können die Vorläuferläsionen des Karzinoms, die Kolonadenome, entdeckt und behandelt werden. In drei europäischen randomisierten Studien konnte gezeigt werden, daß auch die flexible Sigmoidoskopie Inzidenz und Mortalität des kolorektalen Karzinoms um ca. 20\% senken kann. In der vorliegenden Studie, durchgeführt 1993 bis 2001, wurde der Effekt der flexiblen Sigmoidoskopie auf die Inzidenz und Mortalität des kolorektalen Karzinoms untersucht.
In die Interventionsstudie wurden 154900 Männer und Frauen zwischen 55 und 74 Jahren eingeschlossen. Die Teilnehmer erhielten eine flexible Sigmoidoskopie, die nach drei oder fünf Jahren wiederholt wurde. Die durchschnittliche Follow-up-Zeit war 11 Jahre.

Die Mortalität durch das kolorektale Karzinom betrug 2,9/100 000 Personenjahre in der Interventionsgruppe, in der Kontrollgruppe lag die Zahl bei 3,9/100 000 Personenjahre, was einer Risikoreduktion von $26 \%$ entspricht. Die Mortalität des distalen kolorektalen Karzinoms konnte durch die flexible Sigmoidoskopie um 50\% gesenkt werden.

\section{- R. E. Schoen et al.}

Colorectal-Cancer Incidence and Mortality with Screening Flexible Sigmoidoscopy. N Engl J Med 2012; 366: 2345-2357
Kommentar

In dieser großen Interventionsstudie hat die flexible Sigmoidoskopie bewiesen, dass sie in der Lage ist, sowohl die Inzidenz des kolorektalen Karzinoms zu senken als auch die Mortaliät des distalen kolorektalen Karzinoms zu vermindern. Die Inzidenz des proximalen kolorektalen Karzinoms konnte gesenkt werden, weil 21,9\% der untersuchten Patienten eine Koloskopie erhielten. Ein Effekt auf die Mortalität durch ein rechtsseitiges Kolonkarzinom ergab sich dadurch nicht. Die flexible Sigmoidoskopie leistet in dieser Studie mehr als das Screening mit Hilfe des fäkalen okkulten Bluttests, aber wahrscheinlich weniger als die Hälfte im Vergleich zu einer Screening-Koloskopie.

PROF. DR. MED. ReINHARD BÜChSEL .

\title{
Gibt es eine „Löschtaste“ für das Suchtgedächtnis?
}

\begin{abstract}
Gedächtnisinhalte können durch Erinnerung aktiviert, labilisiert und durch geeignete Interventionen auch modifiziert werden, bevor sie sich wieder stabilisieren. Diesen dynamischen Aspekt der Gedächtnisbildung nennt man Rekonsolidierung. Jetzt wurde in einer Studie geprüft, ob dieses Verfahren auch bei opiatabhängigen Patienten wirkt.
\end{abstract}

— 66 opiatabhängigen Patienten wurde nach erfolgreicher Entgiftungstherapie entweder ein neutrales Video oder ein Video mit für den Heroinkonsum relevanten Inhalten gezeigt. Im Anschluss erfolgte eine 60 Minuten dauernde Extinktionssitzung, in dem die Probanden Schlüsselreizen ausgesetzt wurden (Cueexposure). Vor dem Experiment und danach wurden jeweils Cue-induziertes Craving sowie Blutdruck und Puls gemessen.

Das von den Autoren durchgeführte Verfahren verringerte das drogeninduzierte Craving nachhaltig. Das vorlie- gende Experiment stellt möglicherweise einen ersten Ansatz für die Optimierung psychotherapeutischer Verfahren zur Rückfallprävention bei Suchterkrankungen dar.

Als primäres Outcomemaß wurde nur Craving verwendet. Wünschenswert wäre auch gewesen, die Wirksamkeit des Verfahrens auf andere Maße wie zum Beispiel Rückfälle oder drogenfreie Zeit zu untersuchen.

\footnotetext{
- Y.X. Xue et al.

A memory retrieval-extinction procedure to prevent drugcraving and relapse. Science 2012; 336: $241-245$
}

$$
\text { Kommentar }
$$

Kritisch anzumerken ist, dass die Autoren keine Angaben zu den Therapiebedingungen machen. Die Studie wurde im Ankang Hospital und dem Tian-Tang-He Drug Rehabiliatation Center in Peking durchgeführt, Einrichtungen, in denen wohl hauptsächlich Zwangstherapien erfolgen. Inwieweit das Verfahren auf die psychotherapeutischen Versorgung zur Rückfallprävention von Suchtpatienten übertragen werden kann, bleibt abzuwarten, da das Verfahren sich hauptsächlich auf Mechanismen der klassischen Konditionierung konzentriert. Dabei werden motivationale Aspekte von Suchterkrankungen, die auf Einflüsse operanten Konditionierens oder das soziale Umfeld zurückgehen, zu wenig berücksichtigt. DR. MEd. PHILIPPE PFEIFER, MAINZ . 